\title{
ON A CONTINUOUS MAJORITY RULE
}

\author{
WON KYU KIM AND KYOUNG HEE LEE
}

Received 30 March 2005 and in revised form 28 June 2005

\begin{abstract}
A seminal paper by May characterizes the majority rule in terms of anonymity, neutrality, and positive responsiveness. Since then, there have been many characterizations of the majority rule using different axioms by several authors. In this paper, we will introduce the concept of a continuous majority rule which generalizes the previous social welfare functions beyond the discrete values, and using new axioms, we will give a characterization of the continuous majority rule.
\end{abstract}

\section{Introduction}

The problem of the relation between group choice and individual preferences has been stated by Arrow [1] in terms of a "social welfare function." One of the conditions that he puts on this function is that group choice in the presence of two alternatives depends only on individual preferences with respect to this pair of alternatives. The majority rule is an important and reasonable concept to analyze the preference of a society that is to choose between a pair of alternatives. A seminal paper by May [6] characterizes the majority rule in terms of anonymity, neutrality, and positive responsiveness. As remarked in Campbell and Kelly [3], whereas the former two axioms are natural and fairly weak, the positive responsiveness axiom is usually criticized for being too strong. Since then, there have been many characterizations of the majority rule by several authors using several comparable axioms, for example, Pareto optimality, maximal transitivity, reducibility, subsets decomposability, and so forth. However, most of those results dealt with discrete-valued social welfare functions, and so we will need a new continuous majority rule to analyze preferences of the society having continuum values of preferences over two alternatives $x$ and $y$. Also we will need to investigate the fuzzy behavior of preferences of the society having continuum values of preferences over the alternatives. In fact, preferences of the society might change continuously in the process of election campaigning or in the process of negotiation, and changes of public opinion on a special issue are typical examples of fuzzy behavior of preferences. Hence, in a practical point of view, we will need a new continuous concept of the majority rule. 
In this paper, we will introduce a new continuous concept of the majority rule which generalizes previous social welfare functions beyond discrete values, and using new axioms, we will give a characterization of the continuous majority rule. An explicit implication between the majority rule and the continuous majority rule is given in discrete cases. Finally, we will give an interpretation of the continuous majority rule using campaign concepts.

\section{Preliminaries}

Let $x$ and $y$ be two alternatives, and let $S=\{1,2, \ldots, n\}$ denote a society with $n$ individuals called voters. As usual, every voter $i \in S$ has a preference $R_{i} \in\{-1,0,1\}$ over the two alternatives $x$ and $y$. Here $R_{i}=1$ means that voter $i$ prefers $x$ to $y, R_{i}=0$ means that voter $i$ is indifferent, and $R_{i}=-1$ means that voter $i$ prefers $y$ to $x$. As in [9], we recall that the majority rule is the social welfare function MAJ that assigns to any profile $R \in\{-1,0,1\}^{n}$ the sign of aggregate preference $\operatorname{MAJ}(R):=\operatorname{sgn}\left(\sum_{i=1}^{n} R_{i}\right)$ for each $R=$ $\left(R_{1}, \ldots, R_{n}\right) \in\{-1,0,1\}^{n}$. Here, $\operatorname{sgn}(x)$ is the standard sign function for real numbers $x$ with $\operatorname{sgn}(x)=1$ for $x>0, \operatorname{sgn}(x)=0$ for $x=0$, and $\operatorname{sgn}(x)=-1$ for $x<0$.

For a given social welfare function $F: \bigcup_{n>0}\{-1,0,1\}^{n} \rightarrow\{-1,0,1\}$, the following axioms are due to May [6].

Neutrality $(N)$. For any profile $R \in\{-1,0,1\}^{n}$, we have $F(-R)=-F(R)$.

Anonymity (A). For any profiles $R, R^{\prime} \in\{-1,0,1\}^{n}$, where the preferences in $R^{\prime}$ are a permutation of the preferences in $R$, we have $F(R)=F\left(R^{\prime}\right)$.

Positive responsiveness (PR). For any profiles $R, R^{\prime} \in\{-1,0,1\}^{n}$ with $R_{i}^{\prime} \geq R_{i}$ for all $i \in S$ and $R_{j}^{\prime}>R_{j}$ for some $j \in S$, we have that $F(R) \geq 0$ implies $F\left(R^{\prime}\right)=1$. For any profiles $R, R^{\prime} \in\{-1,0,1\}^{n}$ with $R_{i}^{\prime} \leq R_{i}$ for all $i \in S$ and $R_{j}^{\prime}<R_{j}$ for some $j \in S$, we have that $F(R) \leq 0$ implies $F\left(R^{\prime}\right)=-1$.

The above two axioms $(\mathrm{N})$ and $(\mathrm{A})$ are very natural. In fact, the condition $(\mathrm{N})$ states that a welfare function should be indifferent to the alternatives, and the condition (A) states that the aggregate preference should be completely independent of the exact numbering of the voters. However, May's third axiom (PR) is usually criticized as being "too strong" as remarked in [3]. In fact, (PR) requires that whenever the aggregate preference is indifferent, and a single voter changes his/her mind, then the new aggregate preference must follow this single voter.

In 1952, May [6] first proved the following characterization of the majority rule using axioms $(\mathrm{N}),(\mathrm{A})$, and (PR).

Proposition 2.1. A social welfare function $F: \bigcup_{n>0}\{-1,0,1\}^{n} \rightarrow\{-1,0,1\}$ satisfies axioms $(N),(A)$, and $(P R)$ if and only if it is the majority rule MAJ.

Other natural axioms for social welfare functions from the literature are Pareto optimality and weak Pareto optimality as follows.

Pareto optimality (PO). For any profile $R \in\{-1,0,1\}^{n}$ with $R_{i} \geq 0$ for all $i \in S$ and $R_{j}=1$ for some $j \in S$, we have $F(R)=1$. For any profile $R \in\{-1,0,1\}^{n}$ with $R_{i} \leq 0$ for all $i \in S$ and $R_{j}=-1$ for some $j \in S$, we have $F(R)=-1$. 
Weak Pareto optimality (WPO). If $R_{i}=1$ holds for all $i \in S$, then $F(R)=1$. If $R_{i}=-1$ holds for all $i \in S$, then $F(R)=-1$.

PO states that if one alternative is preferred by none of the voters, whereas the other alternative is preferred by some voter, then this other preference should become the aggregate preference. WPO states that if all voters agree, then also the aggregate preference must agree with them. Clearly, PO implies WPO.

Till now, using reasonable axioms, there have been a number of characterizations of the majority rule by several authors (e.g., maximal transitivity (Maskin [5], Dasgupta and Maskin [4]), IIA, limited transitivity, and near symmetry (Campbell and Kelly [3]), PO and weakly path independency (Aşan and Sanver [2]), subsets decomposability (Miroiu [7]), etc.).

In a recent paper [9], Woeginger introduced the another axiom "reducibility to subsocieties" for social welfare functions as follows.

Reducibility to subsocieties (RS). For any profile $R \in\{-1,0,1\}^{n}$ with $n \geq 2$, a social welfare function $M: \bigcup_{n>0}\{-1,0,1\}^{n} \rightarrow\{-1,0,1\}$ satisfies the condition

$$
M(R)=M\left(M\left(R^{-1}\right), M\left(R^{-2}\right), \ldots, M\left(R^{-n}\right)\right),
$$

where $R^{-i}:=\left(R_{1}, \ldots, R_{i-1}, R_{i+1}, \ldots, R_{n}\right)$ for each $n \geq 2$.

When there are exactly 2 voters, then (RS) implies some kind of anonymity.

As remarked in [9], Woeginger gave a reasonable explanation of the naturality of the axiom (RS), and proved another characterization of the majority rule using the axioms $\mathrm{N}, \mathrm{PO}$, and RS without assuming the axioms A and PR as follows.

Proposition 2.2. A social welfare function $M: \bigcup_{n>0}\{-1,0,1\}^{n} \rightarrow\{-1,0,1\}$ satisfies axioms $N, P O$, and $R S$ if and only if it is the majority rule MAJ.

Now we suggest a problem. Given a society of $n$ individuals that is to choose between a pair of alternatives, what is the appropriate method or function that assigns a social preference which can be varied continuously in the continuum set $[-1,1]$ to every profile of individual preferences? For example, during the campaign, the favor of voters continuously changes by effects from new issues, and in many councils and committees, the final decision will be made by complicated negotiation and average opinion of all subsocieties. Hence, for an exact analysis in a mathematical formulation, we will need the following continuum-valued preferences.

Let $x$ and $y$ be two alternatives, and let $S=\{1,2, \ldots, n\}$ denote a society with $n$ individuals called voters. Every voter $i \in S$ has a preference value $R_{i}$ in the continuum set $[-1,1]$ over the two alternatives $x$ and $y$. Here $R_{i}=1$ means that voter $i$ prefers $x$ to $y, R_{i}=0$ means that voter $i$ is indifferent (however it is possible to choose one of the alternatives after a suitable campaign), and $R_{i}=-1$ means that voter $i$ prefers $y$ to $x$ as usual. If $R_{i}$ is a positive value $\alpha \in(0,1)$, then it means that voter $i$ prefers $x$ to $y$ with a positive preference tendency of $\alpha$, and also if $R_{i}$ is a negative value $\beta \in(-1,0)$, then it means that voter $i$ prefers $y$ to $x$ with a positive preference tendency of $-\beta$. Therefore, every voter has a preference value $R_{i}$ in the continuum set $[-1,1]$. If an individual voter is a reasonable person, then the voter might change his/her preference continuously by effects from election campaigns without any drastic changes. In this paper, we will consider the case of 
a continuum-valued social welfare function which gives an aggregate preference for any preference profile of a society.

\section{A continuous majority rule}

As we mentioned before, continuum-valued social welfare functions are very natural in voting systems or public opinion research in the societies, and we should investigate their behaviors and prospects. From now on, for a continuum-valued social welfare function $M$, we may consider every profile vector in the previous axioms $\mathrm{N}, \mathrm{A}, \mathrm{PR}, \mathrm{PO}, \mathrm{WPO}$, and RS as an element of the continuum set $[-1,1]^{n}$ without any confusion.

Let us consider a continuum-valued social welfare function

$$
M: \bigcup_{n>0}[-1,1]^{n} \longrightarrow[-1,1]
$$

Then we are ready to introduce new axioms which are essential in a new characterization of continuum-valued social welfare functions.

Aggregate constancy $(A C)$. For any profile $R=\left(R_{1}, \ldots, R_{n}\right), R^{\prime}=\left(R_{1}^{\prime}, \ldots, R_{n}^{\prime}\right) \in[-1,1]^{n}$ with $\sum_{i=1}^{n} R_{i}=\sum_{i=1}^{n} R_{i}^{\prime}$, we have $M(R)=M\left(R^{\prime}\right)$.

Unanimous decision (UD). For any profile $R=\left(R_{1}, \ldots, R_{n}\right) \in[-1,1]^{n}$ with $R_{1}=R_{2}=$ $\cdots=R_{n}$, we have $M(R)=R_{1}$.

The condition AC clearly satisfies the condition A, and actually AC is a strong condition in contrast to the condition A. However, in real voting situations or in certain organizational committees, when the total sum of preferences of members in the society is the same, the value of aggregate preference of the society is exactly the same, and hence the condition AC makes sense. Also, in discrete cases, AC has almost the same meaning as A. In fact, if the number of zeros are the same in $R$ and $R^{\prime}$, then A and AC are the same. In discrete cases, the condition WPO also coincides with the condition UD.

Here we note that when $S$ has an unanimous opinion, that is, $R_{i}$ has the same value for each $i \in S$, it is plausible to simplify the case so that one of two alternatives $x$ and $y$ should be chosen except for $M(R)=0$. When $S$ has an unanimous opinion with a positive value $\alpha$, then we may assume that $x$ is preferable in the society $S$, and when $S$ has an unanimous opinion with a negative value $\beta$, then we assume that $y$ is preferable in the society $S$.

Even in the discrete case or in the continuum case, our new axiom AC is consistent with the previous axioms PO, PR, and RS for any social welfare function.

We now give some examples showing that there is no implication between the condition $\mathrm{AC}$ and the conditions PR, PO, and RS, respectively.

Example 3.1. (i) The social welfare function $M_{1}: \bigcup_{n>0}[-1,1]^{n} \rightarrow[-1,1]$ is defined by, for each $R=\left(R_{1}, \ldots, R_{n}\right) \in[-1,1]^{n}$,

$$
M_{1}(R):= \begin{cases}1, & \text { if } \forall R_{i} \geq 0, \exists R_{j}=1 ; \\ -1, & \text { if } \forall R_{i} \leq 0, \exists R_{j}=-1 ; \\ 0, & \text { otherwise. }\end{cases}
$$


Then it is easy to see that $M_{1}$ satisfies the conditions $\mathrm{N}$ and PO. However, the conditions AC, PR, RS, and UD are not satisfied by the following examples:

$$
\begin{gathered}
1=M_{1}(1,0,0) \neq M_{1}(1,-1,1)=0 ; \quad 0=M_{1}(0,-1,1)=M_{1}(1,-1,1) \neq 1 ; \\
0=M_{1}(1,1,-1) \neq M_{1}\left(M_{1}(1,-1), M_{1}(1,-1), M_{1}(1,1)\right)=M_{1}(0,0,1)=1 ; \\
0=M_{1}\left(\frac{1}{2}, \frac{1}{2}, \frac{1}{2}\right) \neq \frac{1}{2} .
\end{gathered}
$$

(ii) The social welfare function $M_{2}: \bigcup_{n>0}[-1,1]^{n} \rightarrow[-1,1]$ is defined by

$$
M_{2}(R):=\frac{\sum_{i=1}^{n} R_{i}}{n} \quad \text { for each } R=\left(R_{1}, \ldots, R_{n}\right) \in[-1,1]^{n}
$$

Then we can see that $M_{2}$ satisfies the conditions RS, UD, and AC (by Theorem 3.2 and Remark 3.3). However, the conditions $\mathrm{PO}$ and $\mathrm{PR}$ are not satisfied since $1 / 3=M_{2}(1,0,0)$ and $2 / 3=M_{2}(1,1,0) \neq 1$.

(iii) The majority rule $M_{3}: \bigcup_{n>0}[-1,1]^{n} \rightarrow[-1,1]$, defined by $M_{3}(R):=\operatorname{sgn}\left(\sum_{i=1}^{n} R_{i}\right)$ for each $R=\left(R_{1}, \ldots, R_{n}\right) \in[-1,1]^{n}$, automatically satisfies the axioms AC and PR. However, the conditions UD and RS are not satisfied since $1=M_{3}(1 / 2,1 / 2,1 / 2) \neq 1 / 2$ and $0=$ $M_{3}(1 / 2,1 / 2,-1) \neq M_{3}\left(M_{3}(1 / 2,-1), M_{3}(1 / 2,-1), M_{3}(1 / 2,1 / 2)\right)=M_{3}(-1,-1,1)=-1$.

(iv) The social welfare function $M_{4}: \bigcup_{n>0}[-1,1]^{n} \rightarrow[-1,1]$ is defined by

$$
M_{4}(R):= \begin{cases}\operatorname{sgn}\left(\max _{1 \leq i \leq n} R_{i}\right), & \text { if } R=\left(R_{1}, \ldots, R_{n}\right) \in[-1,1]^{n} \text { with } \forall R_{i} \geq 0, \\ \operatorname{sgn}\left(\min _{1 \leq i \leq n} R_{i}\right), & \text { otherwise. }\end{cases}
$$

Then it is easy to see that $M_{4}$ satisfies the conditions PR and RS. However, the conditions AC and UD are not satisfied since $0=M_{4}(0,0,0) \neq M_{4}(-1,1,0)=-1$, and $1=$ $M_{4}(1 / 2,1 / 2,1 / 2) \neq 1 / 2$.

Next, we will define a new continuous majority rule $M$ which can be suitable for continuum-valued social welfare functions. Let $M: \bigcup_{n>0}[-1,1]^{n} \rightarrow[-1,1]$ be a social welfare function in a society $S=\{1,2, \ldots, n\}$ with $n$ individuals. Then $M$ is called the continuous majority rule if $M$ is the average of aggregate preference for any preference profile of the given society $S$ defined by

$$
M(R):=\frac{\sum_{i=1}^{n} R_{i}}{n} \quad \text { for each } R=\left(R_{1}, \ldots, R_{n}\right) \in[-1,1]^{n}
$$

In fact, $M(R)$ is exactly the arithmetic mean of aggregate preference for a preference profile of a given society $S$, and hence the value $M(R)$ clearly represents the status of the average favor for the alternatives.

Now we will prove a new characterization of the continuous majority rule using axioms AC and UD as follows. 
Theorem 3.2. A continuum-valued social welfare function $M: \bigcup_{n>0}[-1,1]^{n} \rightarrow[-1,1]$ satisfies $A C$ and UD if and only if it is the continuous majority rule.

Proof. One direction of the statement is straightforward. In fact, since the continuous majority rule is defined as the arithmetic mean of aggregate preference, the axioms AC and UD are clearly satisfied.

For the other direction, we will use the inductive argument. When $n=1$, the preference profile $R$ of a given society $S$ is in $[-1,1]$. Then, by the condition $\mathrm{UD}, M(R)=R$. When $n=2$, the preference profile $R=\left(R_{1}, R_{2}\right)$ of a given society $S$ is in $[-1,1]^{2}$. Then, by the conditions AC and UD, we have

$$
M\left(R_{1}, R_{2}\right)=M\left(\frac{R_{1}+R_{2}}{2}, \frac{R_{1}+R_{2}}{2}\right)=\frac{R_{1}+R_{2}}{2} .
$$

We can see that the previous pattern clearly works for every $k \in \mathbb{N}$. In fact, for each preference profile $R=\left(R_{1}, \ldots, R_{k}\right) \in[-1,1]^{k}$, by the conditions AC and UD again, we have

$$
M\left(R_{1}, \ldots, R_{k}\right)=M\left(\sum_{i=1}^{k} \frac{R_{i}}{k}, \ldots, \sum_{i=1}^{k} \frac{R_{i}}{k}\right)=\sum_{i=1}^{k} \frac{R_{i}}{k}
$$

so that the assertion is also true for every $k \in \mathbb{N}$. Therefore, the social welfare function $M$ satisfying the axioms AC and UD must be the continuous majority rule. This completes the proof.

Remarks 3.3. (i) As remarked before, there have been many characterizations of the majority rule using different axioms by several authors as in $[2,3,4,5,6,7,9]$. However, Theorem 3.2 is a simple and new characterization of the majority rule using two natural conditions AC and UD.

(ii) Since the continuous majority rule $M$ is defined as the arithmetic mean of aggregate preference, we can obtain the formula

$$
\begin{aligned}
M\left(M\left(R^{-1}\right), \ldots, M\left(R^{-k}\right)\right) & =M\left(\sum_{i=1, i \neq 1}^{k} \frac{R_{i}}{k-1}, \ldots, \sum_{i=1, i \neq k}^{k} \frac{R_{i}}{k-1}\right) \\
& =M\left(\sum_{i=1}^{k} \frac{R_{i}}{k}, \ldots, \sum_{i=1}^{k} \frac{R_{i}}{k}\right) \\
& =\sum_{i=1}^{k} \frac{R_{i}}{k}=M\left(R_{1}, \ldots, R_{k}\right)=M(R),
\end{aligned}
$$

for each preference profile $R=\left(R_{1}, \ldots, R_{k}\right) \in[-1,1]^{k}$ and all $2 \leq k$, so that the continuous majority rule $M$ satisfies the axiom RS of Woeginger [9]. 
In Theorem 3.2, the axioms AC and UD are very essential so that one cannot drop any of those axioms AC and UD from the statement of Theorem 3.2 without losing the characterization of the continuous majority rule.

Theorem 3.4. There exists a social welfare function $M: \bigcup_{n>0}[-1,1]^{n} \rightarrow[-1,1]$ that is not the continuous majority rule and satisfies the axioms

(a) AC, but not UD;

(b) UD, but not AC.

Proof. For the statement (a), we observe that the social welfare function $M(R) \equiv 0$, for each $R$, satisfies AC, but not UD.

For the statement (b), the following social welfare function $M$ satisfies UD, but not AC:

$$
M(R):= \begin{cases}\frac{\#\left\{i \mid R_{i} \neq 0\right\}}{\sum_{1 \leq i \leq n, R_{i} \neq 0}\left(1 / R_{i}\right)}, & \text { if } \exists R_{i} \neq 0, \sum_{1 \leq i \leq n, R_{i} \neq 0} \frac{1}{R_{i}} \neq 0 \\ 0, & \text { otherwise. }\end{cases}
$$

In fact, $M(R)$ is the harmonic mean of the preferences whenever $R_{i}>0$ for each $1 \leq i \leq n$; and it is clear that $M$ does not satisfy the condition AC since $1=M(1,0) \neq M(1 / 2,1 / 2)=$ $1 / 2$.

Since $\operatorname{sgn}\left(\sum_{i=1}^{n} R_{i}\right)=\operatorname{sgn}\left(\sum_{i=1}^{n} R_{i} / n\right)$ for each $R=\left(R_{1}, \ldots, R_{n}\right) \in[-1,1]^{n}$, we note that the composite function of the continuous majority rule $M$ followed by sgn is actually the same as the majority rule MAJ, that is, $\operatorname{MAJ}(R)=\operatorname{sgn} \circ M(R)$ for each $R \in\{-1,0,1\}^{n}$. Therefore, we can obtain the following which gives an explicit implication between the majority rule and the continuous majority rule in discrete cases.

THeORem 3.5. Let a social welfare function $M: \bigcup_{n>0}[-1,1]^{n} \rightarrow[-1,1]$ satisfy the conditions $A C$ and $U D$. Then the function sgn $\circ M$ defined on the restricted domain $\bigcup_{n>0}\{-1$, $0,1\}^{n}$ is exactly the same as the majority rule MAJ.

\section{Some interpretations and concluding remarks}

Let $x$ and $y$ be two alternatives, and let $S=\{1,2, \ldots, m\}$ be a given society with $m$ individual voters. Every voter $i \in S$ has a preference value $R_{i}$ in the continuum set $[-1,1]$ over the two alternatives $x$ and $y$. For a reasonable interpretation of our continuous majority rule $M(R)$ in real voting systems, we will need some definitions as follows: if $R_{i} \geq 1 / 2$, then we may say that $x$ is strongly preferable on the voter $i$, and if $R_{i} \leq-1 / 2$, then $y$ is called strongly preferable on the voter $i$. These might have reasonable senses in view of real voting situations. In the same line with these, we may say that $x$ is strongly preferable in the society $S$ if at least half of the total voters have strong preferences to $x$.

Then we have the following interpretation of $M(R)$ in case of $|M(R)| \geq 1 / 2$.

Proposition 4.1. Let $M: \bigcup_{m>0}[-1,1]^{m} \rightarrow[-1,1]$ be the continuous majority rule for the given society $S$ with $m$ voters. If $M(R) \geq 1 / 2$ for each profile $R=\left(R_{1}, \ldots, R_{k}\right) \in[-1,1]^{k}$, 
where $k$ is the least integer satisfying $k \geq m / 2$, then $x$ is strongly preferable in the society $S$; and, if $M(R) \leq-1 / 2$ for any profile $R \in[-1,1]^{k}$, then $y$ is strongly preferable in the society $S$.

Proof. It is sufficient to prove the first case. Suppose the contrary, that is, $x$ is not strongly preferable in the society. Then there must exist a preference profile $R^{\prime}=\left(R_{i_{1}}^{\prime}, \ldots, R_{i_{k}}^{\prime}\right)$ such that $R_{i j}^{\prime}<1 / 2$ for each $1 \leq j \leq k$, where $k$ is the least integer satisfying $k \geq m / 2$. Then it is clear that $M\left(R^{\prime}\right)<1 / 2$, which is a contradiction. This completes the proof.

Remark 4.2. In Proposition 4.1, the society $S$ with 3 voters having $\left(R_{1}, R_{2}, R_{3}\right)=(1,1 / 3$, $1 / 3$ ) shows that the condition on $k$ cannot be relaxed. Also, the society $S$ with 4 voters having $\left(R_{1}, R_{2}, R_{3}, R_{4}\right)=(1,1,1 / 3,1 / 3)$ shows that the assumption is not necessary for the strong preferences in the society.

In case of $|M(R)|<1 / 2$, then the situation is more complicated, and the result of voting heavily depends on the campaigns which are suitably designed to the floating voters. For the general theory of voting, see Saari [8] and the references therein.

Concluding remarks. In this paper, we suggest a generalized concept of continuumvalued preference functions which can be used for the continuous majority rule. In discrete cases, the continuum-valued preference functions can be reduced to preference functions for the majority rule due to May [6]. We suggest two new axioms AC and UD which have reasonable meanings both in the discrete case and in the continuous case, and the axiom $\mathrm{AC}$ is shown to be independent of the previous axioms PR, PO, WPO, and RS by using suitable examples. Using the axioms AC and UD, we give a characterization of the continuous majority rule. Also an explicit relation between the majority rule and the continuous majority rule is given in discrete cases. Finally, we give an interpretation of the continuous majority rule using some voting concepts.

\section{Acknowledgment}

The authors would like to thank the referee for the valuable suggestions for the improvement of the paper.

\section{References}

[1] K. J. Arrow, Social Choice and Individual Values, Cowles Commission Monograph, no. 12, John Wiley \& Sons, New York; Chapman \& Hall, London, 1951.

[2] G. Aşan and M. R. Sanver, Another characterization of the majority rule, Econom. Lett. 75 (2002), no. 3, 409-413.

[3] D. E. Campbell and J. S. Kelly, A simple characterization of majority rule, Econom. Theory 15 (2000), no. 3, 689-700.

[4] P. Dasgupta and E. S. Maskin, On the Robustness of Majority Rule, Mimeo, New York, 1998.

[5] E. S. Maskin, Majority rule, social welfare functions, and game forms, Choice, Welfare, and Development (K. Basu, P. K. Pattanaik, and L. Suzumura, eds.), The Clarendon Press, Oxford, 1995, pp. 100-109.

[6] K. O. May, A set of independent necessary and sufficient conditions for simple majority decisions, Econometrica 20 (1952), 680-684.

[7] A. Miroiu, Characterizing majority rule: from profiles to societies, Econom. Lett. 85 (2004), no. 3, 359-363. 
[8] D. G. Saari, Unsettling aspects of voting theory, Econom. Theory 22 (2003), no. 3, 529-555.

[9] G. J. Woeginger, A new characterization of the majority rule, Econom. Lett. 81 (2003), no. 1, 89-94.

Won Kyu Kim: Department of Mathematics Education, Chungbuk National University, Cheongju 361-763, Korea

E-mail address: wkkim@chungbuk.ac.kr

Kyoung Hee Lee: Division of Liberal Arts, Korea University of Technology \& Education, Chunan 330-708, Korea

E-mail address: khlee@kut.ac.kr 


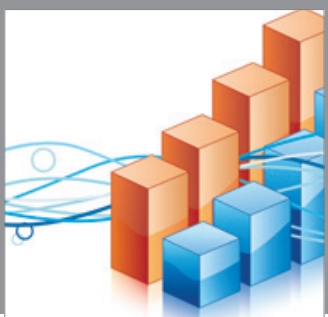

Advances in

Operations Research

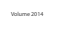

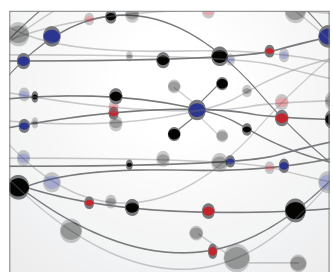

\section{The Scientific} World Journal
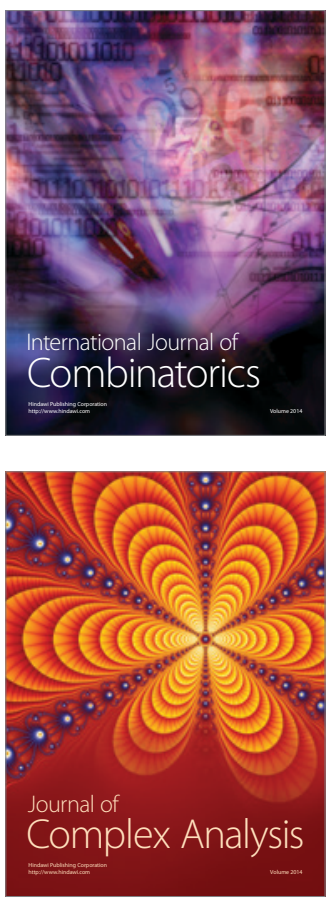

International Journal of

Mathematics and

Mathematical

Sciences
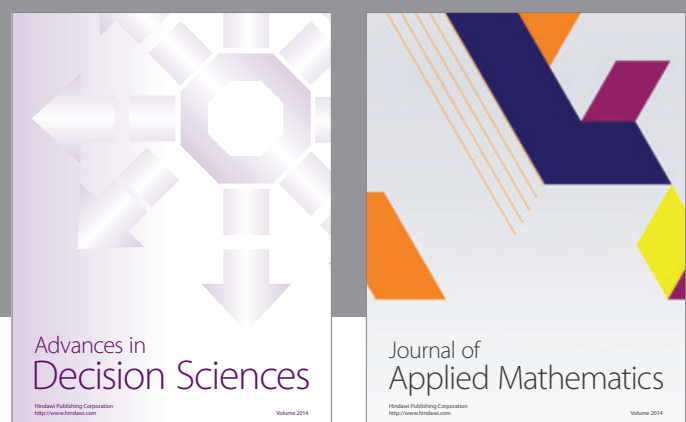

Journal of

Applied Mathematics
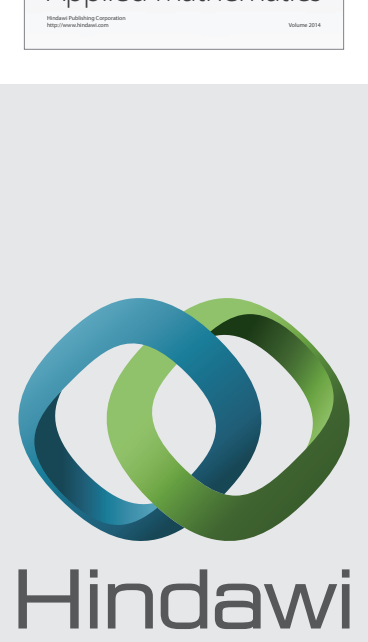

Submit your manuscripts at http://www.hindawi.com
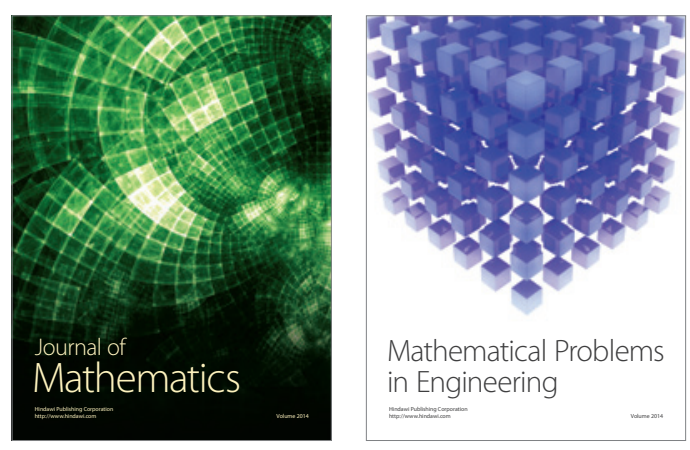

Mathematical Problems in Engineering
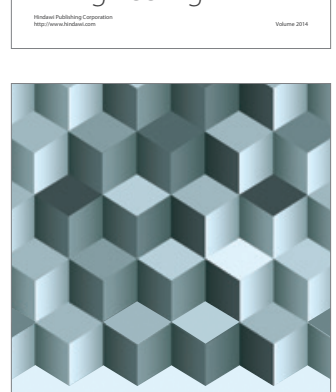

Journal of

Function Spaces
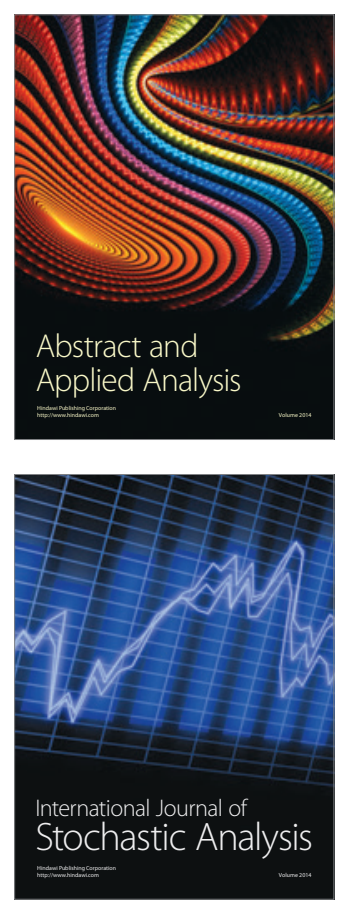

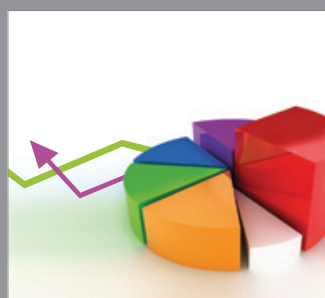

ournal of

Probability and Statistics

Promensencen
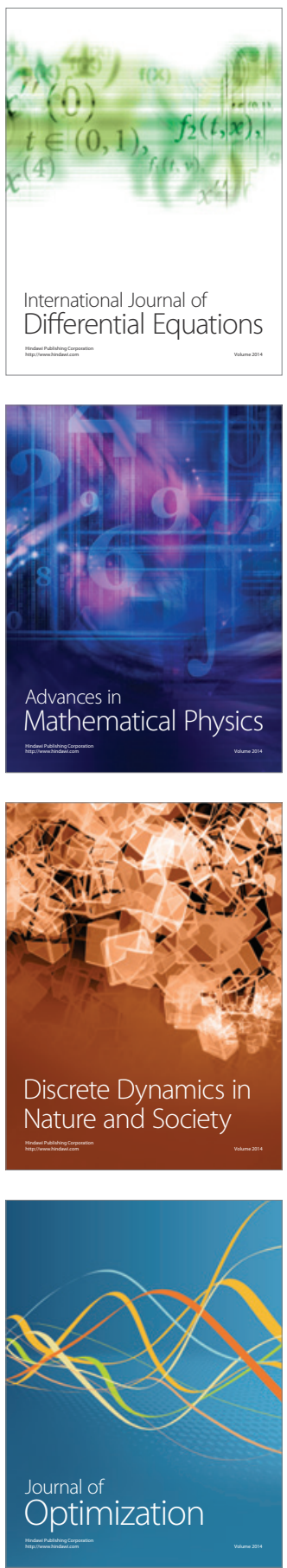